\title{
White Matter Abnormality Correlates with Developmental and Seizure Outcomes in West Syndrome of Unknown Etiology
}

(D). Natsume, (D). Ogawa, (D). Fukasawa, (D). Yamamoto, (D). Ishihara, (D). Sakaguchi, (D). Ito, (D). Takeuchi, (D). Azuma, (D) N. Ando, (D)T. Kubota, (D). Tsuji, (DH. Kawai, DS. Naganawa, and DH. Kidokoro

\begin{abstract}
BACKGROUND AND PURPOSE: West syndrome is an epileptic encephalopathy characterized by epileptic spasms, a specific pattern on electroencephalography of hypsarrhythmia, and developmental regression. Our aim was to assess white matter abnormalities in West syndrome of unknown etiology. We hypothesized that diffusion tensor imaging reveals white matter abnormalities, especially in patients with poor seizure and developmental outcomes.
\end{abstract}

MATERIALS AND METHODS: We enrolled 23 patients with new-onset West syndrome of unknown etiology. DTI was performed at 12 and 24 months of age. Fractional anisotropy images were compared with those of controls by using tract-based spatial statistics. We compared axial, radial, and mean diffusivity between patients and controls in the fractional anisotropy skeleton. We determined correlations of these parameters with developmental quotient, electroencephalography, and seizure outcomes. We also compared DTI with hypometabolism on fluorodeoxyglucose positron-emission tomography.

RESULTS: At 12 months of age, patients showed widespread fractional anisotropy reductions and higher radial diffusivity in the fractional anisotropy skeleton with a significant difference on tract-based spatial statistics. The developmental quotient at 12 months of age correlated positively with fractional anisotropy and negatively with radial and mean diffusivity. Patients with seizure and abnormal findings on electroencephalography after initial treatments had lower fractional anisotropy and higher radial diffusivity. At 24 months, although tract-based spatial statistics did not show significant differences between patients and controls, tract-based spatial statistics in the 10 patients with a developmental quotient of $<70$ had significant fractional anisotropy reduction. In patients with unilateral temporal lobe hypometabolism on PET, tract-based spatial statistics showed greater fractional anisotropy reduction in the temporal lobe ipsilateral to the side of PET hypometabolism.

CONCLUSIONS: Diffuse abnormal findings on DTI at 12 months of age suggest delayed myelination as a key factor underlying abnormal findings on DTI. Conversely, asymmetric abnormal findings on DTI at 24 months may reflect underlying focal pathologies.

ABBREVIATIONS: $\mathrm{ACTH}=$ adrenocorticotropic hormone; $\mathrm{DQ}=$ developmental quotient; $\mathrm{EEG}=$ electroencephalography; $\mathrm{FA}=$ fractional anisotropy; TBSS $=$ tract-based spatial statistics; WS $=$ West syndrome

W est syndrome (WS) is an epileptic encephalopathy characterized by epileptic spasms, a specific pattern on electroencephalography (EEG) of hypsarrhythmia, and developmental re-

Received June 16, 2015; accepted after revision August 26.

From the Departments of Pediatrics (J.N., C.O., H.Y., N.I., Y.S., Y.I., T. Takeuchi, Y.A. H. Kidokoro), Developmental Disability Medicine (J.N.), and Radiology (H. Kawai, S.N.), Nagoya University Graduate School of Medicine, Nagoya, Japan; Department of Pediatrics and Neonatology (N.A.), Nagoya City University Graduate School of Medical Sciences, Nagoya, Japan; Brain and Mind Research Center (J.N., H. Kidokoro), Nagoya University, Nagoya, Japan; Department of Pediatrics (T.F., T.K.), Anjo Kosei Hospital, Anjo, Japan; and Department of Pediatrics (T. Tsuji), Okazaki City Hospital, Okazaki, Japan.

This study was supported by a Grant-in-Aid for Scientific Research (C) 23591492 and a Grant-in-Aid for Scientific Research on Innovative Areas (Comprehensive Brain Science Network) from the Ministry of Education, Culture, Sports, Science and Technology of Japan. gression. While WS has a variety of causes, the etiology remains unclear in $20 \%$ of patients. ${ }^{1}$ Recent advances in neuroimaging have revealed underlying pathologies such as focal cortical dysplasia in these patients. ${ }^{2} \mathrm{We}$ and others have reported hypometabolism on PET and hypoperfusion on SPECT during the early stages of cryptogenic WS. ${ }^{3-9}$ Detecting these findings is useful for both predicting long-term outcomes and detecting underlying lesions. ${ }^{3-6,8,9}$ Further progress of modern neuroimaging techniques

\footnotetext{
The authors have no financial relationships relevant to this article to disclose or no conflicts of interest to disclose.

Please address correspondence to Jun Natsume, MD, PhD, Department of Pediatrics, Nagoya University Graduate School of Medicine, 65 Tsurumai-cho, Showa-ku, Nagoya, Aichi 466-8550, Japan; e-mail: junnatsu@med.nagoya-u.ac.jp

-- Indicates open access to non-subscribers at www.ajnr.org

http://dx.doi.org/10.3174/ajnr.A4589
} 
is expected to clarify the underlying pathology in patients with WS of unknown etiology.

Diffusion tensor imaging is an MR imaging technique that can provide information about the orientation and integrity of white matter fibers. This method is useful for detecting underlying abnormalities in the white matter of patients with various neurologic disorders. Because it is hypothesized that widespread networks of cortical and subcortical structures are involved in the generation of epileptic spasms and hypsarrhythmia in WS, ${ }^{10}$ DTI should help reveal the pathophysiology of this syndrome.

In this prospective study, we performed serial DTI studies from the onset of epilepsy to assess underlying white matter abnormalities in patients with WS of unknown etiology. We compared the results of DTI with cognitive and motor development, EEG findings, and seizure outcomes. We also compared distributions of abnormal findings on DTI with those of hypometabolism on $\left[{ }^{18} \mathrm{~F}\right]$ fluorodeoxyglucose-PET.

\section{MATERIALS AND METHODS}

This study was approved by the research ethics committee at Nagoya University Graduate School of Medicine.

\section{Patients}

Between 2007 and 2014, we performed DTI in consecutive patients with new-onset WS of unknown etiology seen in Nagoya University Hospital. We diagnosed WS from epileptic spasms in clusters that were recorded by simultaneous video-EEG recording and typical interictal EEG findings of hypsarrhythmia. Inclusion criteria for patients were as follows: 1) normal birth and absence of any etiologic factors related to WS, 2) normal development before the onset of spasms and the absence of neurologic abnormalities at onset, 3) occurrence of spasm clusters without any other types of seizures before the onset of spasms, and 4) normal findings from laboratory tests, CT, and MR imaging. Chromosomal abnormalities were investigated with G-banding or array comparative genomic hybridization.

Patients were initially treated according to the following protocol: Clonazepam was administered for 1 week. When other oral antiepileptic drugs were started in previous hospitals, they were continued for 1 week. If oral antiepileptic medications failed to control the spasms, adrenocorticotropic hormone (ACTH) therapy was started. In the present study, 1 patient did not receive ACTH therapy. In ACTH therapy, synthetic ACTH was injected intramuscularly at $0.015 \mathrm{mg} / \mathrm{kg} /$ day for 2-4 weeks, followed by alternate-day administration for 1 week. The effectiveness of each drug was evaluated on the basis of the frequency of spasms and interictal EEG findings.

\section{Controls}

We used 2 control groups to compare the images of patients with WS. The first control group consisted of 13 children with a mean age of $12.2 \pm 3.0$ months (range, 8-18 months) and was used for the analysis of patients at 12 month of age. The second control group comprised 15 children with a mean age of $24.8 \pm 4.5$ months (range, 18-27 months) and was used for the analysis of patients at 24 months. Controls had undergone MR imaging because CNS abnormalities had been suspected on presentation but were later ruled out. Conventional MR imaging did not show any abnormalities in controls. Final diagnoses in controls were nonpathologic sleep myoclonus, nonpathologic motion arrest, myasthenia gravis, pigmented macules without neurocutaneous disorders, peripheral facial nerve palsy, breath-holding spell, shuddering attack, nonpathologic macrocephaly, and short stature.

\section{Image Acquisition for DTI}

All patients underwent DTI 3 times. The first scans were obtained at the onset of spasms before ACTH therapy; the second, at 12 months of age; and the third, at 24 months of age. We used the second and third scans for the present study. The second and third scans were obtained $>1$ month after the end of ACTH therapy to avoid the effects of brain shrinkage caused by ACTH. In our previous study evaluating MR imaging by visual inspection, we did not detect cerebral shrinkage caused by ACTH therapy on MR imaging performed $>1$ month after the end of ACTH therapy. ${ }^{5}$ Scans were obtained as part of the clinical routine to search for underlying pathology. Before each scan, we explained to parents that the results would be used for research, and informed consent was obtained. All patients were sedated with oral chloral hydrate before the examination. When patients did not appear sufficiently sedated after chloral hydrate intake, intravenous midazolam or ketamine was administered.

MR imaging examination was performed on a 3T scanner (Magnetom Trio A Tim System; Siemens, Erlangen, Germany) with a 32-channel phased array head coil. DTI parameters were the following: 12 noncollinear diffusion directions (b-value = $1000 \mathrm{~s} / \mathrm{mm}^{2}$ ) with a nondiffusion gradient $\left(\mathrm{b}\right.$-value $\left.=0 \mathrm{~s} / \mathrm{mm}^{2}\right)$; TR, $7800 \mathrm{~ms}$; TE, $84 \mathrm{~ms}$; FOV, $269 \times 269 \mathrm{~mm}$; matrix, $128 \times 128$; and 64 axial sections (voxel size $=2.1 \times 2.1 \times 2.1 \mathrm{~mm}^{3}$ ). In addition to DTI data, the following conventional MR images were acquired to examine structural abnormalities: axial T1-weighted, T2-weighted, fluid-attenuated inversion recovery images, sagittal T1-weighted images, and coronal T2-weighted images.

\section{Evaluation of EEG, Seizure, and Neurodevelopmental Outcomes}

We used EEG findings at 12 months of age for evaluating the early response to initial treatments. EEG findings at this age were classified as normal or abnormal on the basis of the remaining epileptiform discharges during wakefulness and sleep. Evaluation of seizure outcome was performed between August and December 2014. The age at the evaluation ranged from 2 to 8 years (median, 5 years). The information was obtained from the medical charts at Nagoya University or affiliated hospitals. Seizure outcome was categorized as follows: 1) free of seizures, when the patient had no seizures during the entire follow-up after the initial treatment with clonazepam or ACTH; or 2) persistent or recurrent seizures during the entire follow-up after initial treatment.

The developmental quotient (DQ) was estimated by using the Tsumori-Inage developmental questionnaire at 12 and 24 months of age. ${ }^{11}$ This questionnaire was released in 1961 and is widely used in Japan. ${ }^{9,12}$ It consists of the 5 domains of gross motor skill, fine motor skill, sociality, activities of daily living, and language. Developmental status was considered as follows: 1) normal, when 
patients had a DQ of $\geq 70$ at 24 months of age; or 2) delayed cognitive and motor development, when patients had a DQ of $<70$ at 24 months of age. While the DQ at 12 months tends to reflect development of motor function, the DQ at 24 months reflects language and cognitive function more than the one at 12 months.

\section{FDG-PET}

All patients underwent FDG-PET at onset and at 12 and 24 months of age. The scans were obtained to search for underlying pathologies and predict long-term outcome as in our previous studies. ${ }^{5,9}$ All patients were sedated by chloral hydrate suppository during the PET examination. PET images were analyzed with visual inspection by 3 pediatric neurologists (J.N., T.F., and H. Kidokoro). All 3 pediatric neurologists were regularly involved in neuroimaging studies and had experience in the interpretation of pediatric PET images. PET findings were evaluated as in our previous studies. ${ }^{5,9}$ Regional hypometabolism was defined as a regional decrease in FDG accumulation in $\geq 2$ gyri on $\geq 2$ sections.

\section{Image Processing}

DTI analyses were performed by using tract-based spatial statistics (TBSS) in the fMRI of the Brain software package (FSL; http:// www.fmrib.ox.ac.uk/fsl). ${ }^{13}$ For each subject, all DTI was corrected for eddy current-induced distortion and subject motion effects. The correction was performed by affine registration to a reference volume that is included in the FMRIB Diffusion Toolbox (http://fsl.fmrib.ox.ac.uk/fsl/fslwiki/FDT).

A brain mask was created from the first B0 image. A diffusion tensor model at each voxel was fitted, and fractional anisotropy (FA), mean diffusivity, axial diffusivity, and radial diffusivity maps were computed. Next, the most representative FA image was first identified, and all FA data for a subject were aligned to this target image by nonlinear registration. The mean FA image was created and thinned to create a mean FA skeleton, which represents the centers of all tracts common to the group. Aligned FA data for each subject were then projected onto this skeleton, and the resulting data were fed into voxelwise cross-subject statistics. Statistical analysis was performed voxel by voxel to detect regions of significant differences in FA between the 2 groups of subjects, by using FSL Randomise (http://fsl.fmrib.ox.ac.uk/fsl/ fslwiki/Randomise) with 5000 permutations. The Threshold-Free Cluster Enhancement option in Randomise was used to avoid an arbitrary initial cluster-forming threshold. Age was used as a nuisance covariate of no interest. Results are reported at a corrected threshold of $P<.05$. TBSS analysis was performed between FA images of all patients and controls. Analyses were also performed between subgroups of patients with a DQ of $<70$ or a DQ of $\geq 70$ at 12 and 24 months of age and controls.

Skeletons showing significantly reduced FA from those of controls at 12 months of age were extracted. These skeletons included whole clusters with significant reduction of FA in patients on TBSS analysis and did not include clusters of increased FA. Mean values for FA, mean diffusivity, and eigenvalues in the skeletons were calculated in each patient. Eigenvalues $(\lambda 1, \lambda 2, \lambda 3)$ correspond to the directional apparent diffusion coefficients as either principal axial diffusivity $(\lambda 1)$ or radial diffusivity $([\lambda 2+\lambda 3] / 2)$.

\section{Statistical Analysis}

Statistical analysis was performed by using SPSS, Version 19 software (IBM, Armonk, New York). We compared mean DTI parameters in the skeleton with significant differences on TBSS between patients and controls and determined correlations of parameters with the DQ at 12 months of age in patients. We also compared DTI parameters between patients with and without abnormal findings on EEG at 12 months of age and between patients with and without persisting or relapsing seizures after initial treatment.

On the basis of FDG-PET findings at 12 and 24 months of age, TBSS analysis of DTI at 12 and 24 months of age was performed between patients with regional hypometabolism and controls and between patients without regional hypometabolism and controls. This evaluation was performed because patients with regional hypometabolism may have focal underlying pathology that causes regional abnormal findings on DTI.

Comparisons of DTI parameters between patients and controls, between patients with and without EEG abnormalities, and between patients with and without persisting or relapsing seizures were performed by the nonparametric Mann-Whitney $U$ test or the Kruskal-Wallis test and post hoc analysis with Bonferroni correction for analysis among 3 groups of patients with and without seizures and controls, and those with normal or abnormal EEG findings and controls. Correlations of parameters in patients with DQ were determined by the Spearman correlation coefficient $(r)$. Significance was established at $P<.05$.

\section{RESULTS}

Twenty-three patients (14 boys) with WS of unknown etiology underwent DTI during the study period. ACTH therapy was performed as an initial treatment in 22 patients, while the remaining patient did not receive ACTH therapy. The interval from the onset of spasms to initial treatment was $0-4$ months (median, 1 month). After initial treatment, 15 patients were categorized as free of seizures; 6 patients experienced recurrence of spasms; and 2 patients showed partial seizures. EEG at 12 months of age showed normal findings in 11 patients, hypsarrhythmia in 3, and focal spikes in 9. In 21 of 23 patients, the presence or absence of abnormal findings on EEG was the same at 12 and 24 months of age. In 1 patient with hypsarrhythmia and 1 with focal spikes at 12 months of age, the EEG findings were normal at 24 months. In 15 patients free of seizures after initial treatment, the EEG findings at 12 months of age were normal in 10 patients and showed focal spikes in 5 . The DQ ranged from 33 to 100 (median, 77) at 12 months and from 29 to 123 (median, 75 ) at 24 months of age.

\section{TBSS Analysis between Patients and Controls}

Two patients could not undergo DTI at 12 months of age, and DTI of another patient showed motion artifacts. TBSS analysis at 12 months was thus performed between 20 patients and 13 controls. TBSS revealed reduced FA in the corpus callosum, 

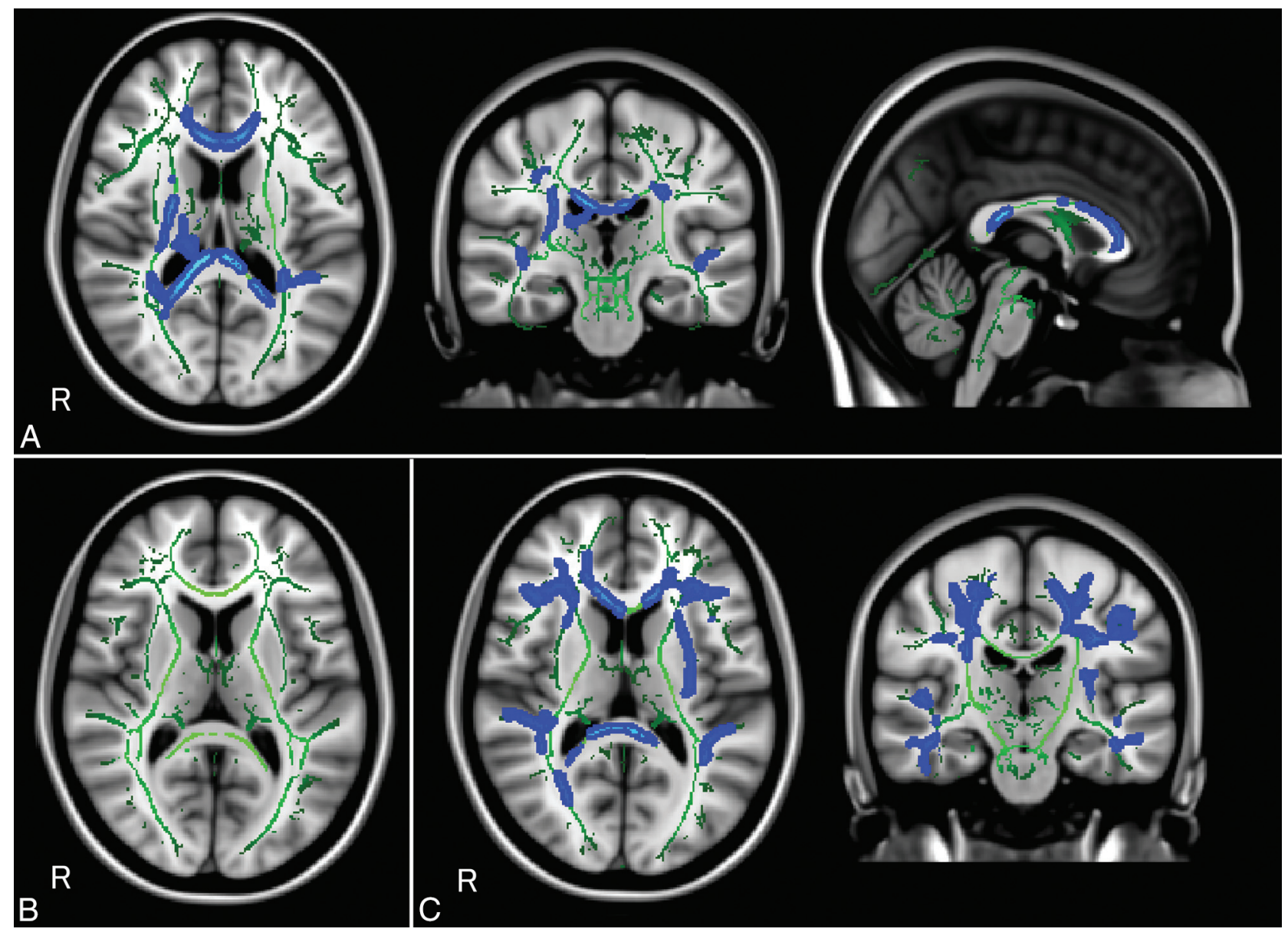

FIG 1. Tract-based spatial statistics analysis between patients and controls at 12 and 24 months of age. A, Tract-based spatial statistics at 12 months of age reveals reduced fractional anisotropy values (blue areas) in the corpus callosum, deep white matter, and subcortical white matter in the temporal lobes of patients. The green areas represent white matter skeletons with a threshold FA of $\geq 0.2$. B, Tract-based spatial statistics at 24 months of age shows no significant difference between all patients and controls. $C$, Tract-based spatial statistics reveals reduced fractional anisotropy values (blue areas) in widespread white matter areas of patients with a developmental quotient of $<70$ at 24 months of age.

Mean values of diffusion tensor imaging parameters in FA skeletons with a significant difference on tract-based spatial statistics at 12 months of age ${ }^{a}$

\begin{tabular}{lccc}
\hline & Patients $(\boldsymbol{n}=\mathbf{2 0})$ & Controls $(\boldsymbol{n}=13)$ & $\boldsymbol{P}$ \\
\hline FA & $0.46 \pm 0.04$ & $0.51 \pm 0.02$ & $<.001$ \\
$\mathrm{AD}, \lambda 1\left(\mathrm{~mm}^{2} / \mathrm{s}\right)$ & $0.0016 \pm 0.0001$ & $0.0016 \pm 0.0001$ & NS \\
$\mathrm{RD},(\lambda 2+\lambda 3) /$ & $0.00074 \pm 0.00007$ & $0.00066 \pm 0.00006$ & .007 \\
$2\left(\mathrm{~mm}^{2} / \mathrm{s}\right)$ & & &
\end{tabular}

$\begin{array}{lll}\mathrm{MD}\left(\mathrm{mm}^{2} / \mathrm{s}\right) & 0.0010 \pm 0.0001 & 0.0010 \pm 0.0007 \quad \mathrm{NS}\end{array}$

Note:-AD indicates axial diffusivity; MD, mean diffusivity; NS, not significant; RD, radial diffusivity

${ }^{\text {a }}$ Statistical analysis was performed with a nonparametric Mann-Whitney $U$ test.

deep white matter, and subcortical white matter in the temporal lobes of patients (Fig $1 A$ ). No areas of increased FA were identified.

DTI of 3 patients at 24 months of age showed motion artifacts, so DTI of the remaining 20 patients was used for analysis. TBSS at 24 months of age did not show any significant difference between all patients and controls (Fig $1 B$ ). Comparisons between the 10 patients with a DQ of $<70$ at 24 months of age and controls revealed reduced $F A$ values in widespread white matter in patients (Fig 1C). No areas showed significantly different FA between patients with a DQ of $\geq 70$ and controls.

\section{Parameters in the FA Skeleton with Significant Differences on TBSS and Developmental or Seizure \\ Outcomes}

Mean values of FA, mean diffusivity, and eigenvalues in the FA skeleton showing significant FA reduction in patients on TBSS at 12 months of age are shown in the Table. Axial diffusivity along the axonal direction $\lambda 1$ and mean diffusivity showed no significant difference between patients and controls. Radial diffusivity perpendicular to the axonal direction $([\lambda 2+\lambda 3] / 2)$ was significantly higher in patients $(P=.007)$.

The DQ at 12 months of age showed a significant positive correlation with FA $(r=0.64, P=.003)$ and negative correlations with radial and mean diffusivity $(r=-0.60, P=.005$ and $r=-0.53, P=.017$, respectively) (Fig 2 ). No correlation was identified between axonal diffusivity $\lambda 1$ and DQ at 12 months of age.

Patients with seizures after initial treatment showed significant decreases in FA compared with patients without seizures and with controls (mean FA: $0.42 \pm 0.03$ versus $0.47 \pm 0.03, P=.019$, versus $0.51 \pm 0.02, P<.001$, by post hoc analysis with Bonferroni correction, respectively) and increased radial diffusivity compared with patients without seizures and with controls (mean $[\lambda 2+\lambda 3] / 2: 0.00081 \pm 0.00005 \mathrm{~mm}^{2} / \mathrm{s}$ versus $0.00071 \pm 0.00006$ 

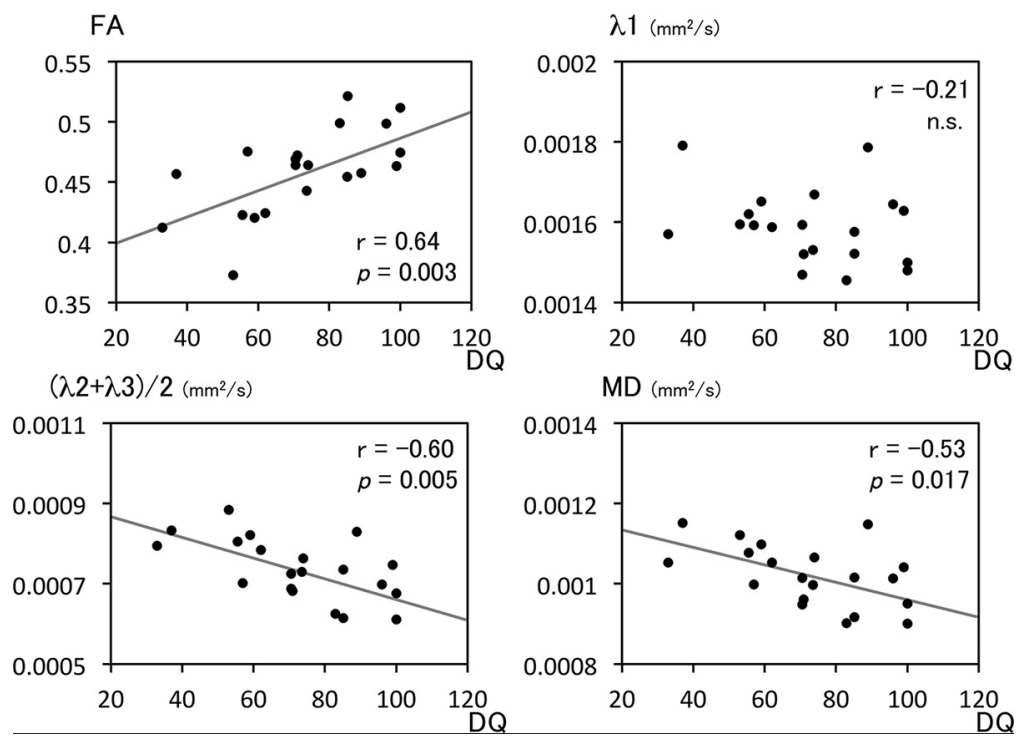

FIG 2. Correlation of the developmental quotient and diffusion tensor imaging parameters at 12 months of age. The developmental quotient at 12 months of age shows a significant positive correlation with fractional anisotropy and negative correlations with radial $([\lambda 2+\lambda 3] / 2)$ and mean diffusivity. No correlation is evident between axonal diffusivity $(\lambda 1)$ and the developmental quotient at 12 months of age.

FA

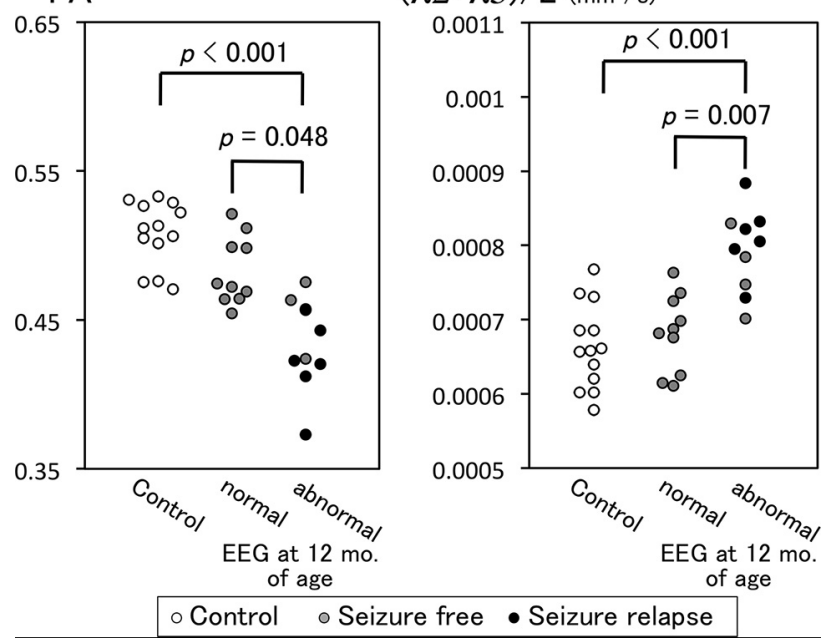

FIG 3. Abnormal findings on EEG at 12 months of age and diffusion tensor imaging parameters. Decreases in fractional anisotropy and increases in radial diffusivity are observed in patients with abnormalities findings on EEG compared with patients with normal electroencephalography findings at 12 months of age. White dots represent controls, gray dots are patients without seizures after initial treatments, and black dots are patients with relapsing or persistent seizures. Patients with relapsing or persistent seizures have low FA and high radial diffusivity.

$\mathrm{mm}^{2} / \mathrm{s}, P=.046$, versus $0.00066 \pm 0.00006 \mathrm{~mm}^{2} / \mathrm{s}, P=.001$, by post hoc analysis with Bonferroni correction, respectively). Decreased FA and increased radial diffusivity were observed in patients with abnormal findings on EEG at 12 months of age compared with patients with normal findings on EEG at 12 months of age and with controls (mean FA: $0.43 \pm 0.03$ versus $0.48 \pm 0.02$, $P=.048$, versus $0.51 \pm 0.02, P<.001$; mean $[\lambda 2+\lambda 3] / 2$ : $0.00079 \pm 0.00005 \mathrm{~mm}^{2} / \mathrm{s}$ versus $0.00068 \pm 0.00005 \mathrm{~mm}^{2} / \mathrm{s}, P=$ .007 , versus $0.00066 \pm 0.00006 \mathrm{~mm}^{2} / \mathrm{s}, P<.001$, by post hoc analysis with Bonferroni correction, respectively) (Fig 3). No sig- nificant differences in $\lambda 1$ were seen between patients with and without seizures or with and without EEG abnormality at 12 months of age.

\section{Correlation of DTI and FDG-PET}

FDG-PET at 12 months of age showed regional cortical hypometabolism in 16 patients. The regions of hypometabolism at 12 months were unilateral temporal in 3 patients, unilateral frontal in 2 , unilateral parietal in 1 , unilateral frontotemporal in 6, unilateral parietotemporal in 2, unilateral temporooccipital in 1, and unilateral frontoparieto-temporal in 1. FDG-PET at 24 months of age revealed regional cortical hypometabolism in 8 patients and normal findings in the other 12 patients on visual inspection. Of 16 patients with PET hypometabolism at 12 months, 6 had relapse or persistent seizures and 10 had abnormal findings on EEG at 12 months. Of 8 patients with PET hypometabolism at 24 months, 5 had seizure relapse or persistent seizures and 5 had abnormal findings on EEG at 24 months. At 12 months of age, hypometabolism was on the left side in 10 patients, the right side in 5 , and in the bilateral temporal lobes in 1 . At 24 months of age, hypometabolism was present in the left temporo-frontal lobe in 3 patients, left temporal lobe in 1 , right temporo-frontal lobe in 2 , right frontal lobe in 1 , and right temporal lobe and right frontal lobe in 1. FA images of patients with right-sided hypometabolism were flipped, so that all regional hypometabolism would appear on the left side. We excluded 1 patient with hypometabolism in the bilateral temporal lobes to compare the laterality of the abnormality on PET and DTI and 2 patients with right or left frontal hypometabolism on TBSS analysis to focus on temporal lobe hypometabolism. At 12 months of age, TBSS between the 15 patients with left temporal hypometabolism and controls showed reduced FA in widespread white matter symmetrically. At 24 months of age, TBSS between the 7 patients with left temporal hypometabolism and controls showed reduced FA in widespread white matter and more prominent hypometabolism in the left temporal lobe compared with the right temporal lobe (Fig 4). TBSS did not show FA reduction in 12 patients without PET hypometabolism at 24 months of age compared with controls.

\section{DISCUSSION}

DTI in patients with WS of unknown etiology showed widespread white matter abnormalities at 12 months of age. While axial diffusivity showed no significant differences compared with controls, radial diffusivity was significantly increased. The DQ correlated positively with FA and negatively with radial or mean diffusivity. Patients with persistent EEG abnormality and seizures had more prominent DTI abnormalities than patients with normal EEG and no seizures after initial treatment. Although DTI at 24 months of age did not show significant differences in the com- 

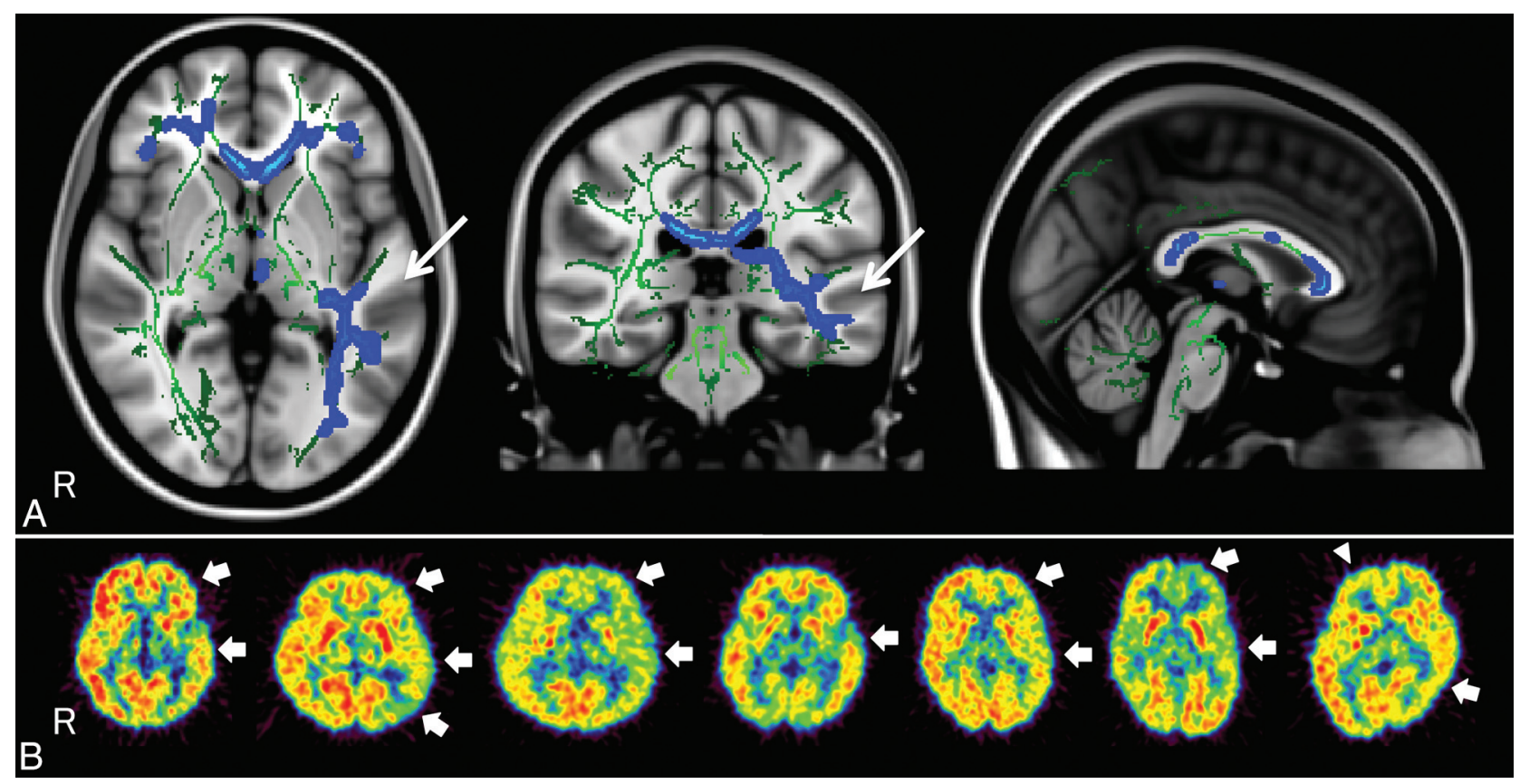

FIG 4. Comparison of tract-based spatial statistics among patients with left temporal hypometabolism on positron-emission tomography at 24 months of age. A, Comparison of tract-based spatial statistics among 7 patients with left temporal hypometabolism and controls shows reduced fractional anisotropy in widespread white matter (blue areas) and more prominent DTI abnormalities in the left temporal lobe (arrows) compared with the right one. The green areas represent white matter skeletons with a threshold FA of $\geq 0.2$. B, Fluorodeoxyglucose positronemission tomography of 7 patients. Positron-emission tomography shows left temporal or temporofrontal hypometabolism (arrows). Note that the images from 3 patients with right temporal lobe hypometabolism are flipped, so all areas of hypometabolism are shown on the left side. The last patient shows additional hypometabolism in the contralateral frontal lobe (arrowhead).

parisons of all patients and controls, patients with poor developmental outcomes still showed decreased FA. Patients with lateralized regional hypometabolism on PET showed more prominent DTI abnormalities ipsilateral to the side of hypometabolism. This is the first report revealing abnormal findings on DTI in patients with WS of unknown etiology. A recently published study by Fosi et $\mathrm{al}^{14}$ did not show a significant difference between patients with WS and controls on TBSS analysis of DTI. The timing of MR imaging scans in their study was not clearly described and seems to be variable. The standardized timing of MR imaging in our study may be one of the reasons that MRI revealed significant abnormality.

Several factors can cause abnormal findings on DTI, such as axonal properties, fiber coherence, demyelination, and white matter maturation, especially in infants. ${ }^{15-17}$ Cortical malformation often affects the underlying white matter and the cortex ${ }^{18}$ and could disrupt white matter tracts and cause reductions in anisotropy. ${ }^{19,20}$ White matter abnormality could also be attributed to propagation of epileptic activities along white matter tracts. ${ }^{21}$ ACTH therapy also possibly affects white matter properties, though we performed DTI $>1$ month after the end of ACTH therapy to avoid the effects of ACTH. Another major factor contributing to DTI changes in infancy is white matter maturation. In this period, age-related changes in water diffusion-based properties are observed in white matter tracts. ${ }^{17,22-26} \mathrm{We}$ have reported delayed myelination on T2-weighted imaging at approximately 10 months of age in patients with cryptogenic WS. ${ }^{5}$

In an unpublished study of ours, a significant decrease of FA was revealed in patients with delayed myelination on T2WI at 12 months of age. The diffuse abnormal findings on DTI in the pres- ent study were prominent at 12 months of age and had resolved by 24 months of age in patients with good developmental outcomes. Dynamic changes in abnormal findings on DTI resemble the pattern of delayed myelination in our previous study of WS, suggesting that delayed white matter maturation may contribute to the abnormal findings on DTI. Furthermore, FA reduction in our study was attributed to increased radial diffusion perpendicular to the axonal direction. Increased radial diffusion suggests abnormal myelination in experimental models, though pathologic correlates of abnormal findings on DTI in humans are still unclear. ${ }^{15,16}$ Given the dynamic changes in abnormal findings on DTI and the increased radial direction, delayed myelination may be the most likely pathology underlying widespread symmetric abnormal findings on DTI at 12 months of age.

A strong correlation was observed between DTI parameters and cognitive and motor development in our study. Correlations between DTI and cognitive function have been reported in healthy children and those with various pediatric disorders. Widjaja et $\mathrm{al}^{27}$ studied correlations between abnormal findings on DTI and neuropsychological function in children with nonlesional focal epilepsy, revealing that widespread white matter abnormalities were associated with impaired neuropsychological function. The impairment in white matter may reflect disruption in the connectivity of cortical processing networks, which is necessary for the development of cognition. ${ }^{27}$ In our study, delayed maturation of widespread white matter areas contributed to delayed cognitive and motor development. This abnormal white matter included many fiber tracts that are important for language and cognitive function, such as the uncinate fasciculus and inferior 
longitudinal fasciculus. Evaluation of DTI can thus facilitate objective assessment of the developmental status in a patient with WS.

We revealed a difference in DTI parameters between patients with and without abnormal findings on EEG and those with and without seizures after treatment. Juhász et $\mathrm{al}^{10}$ hypothesized that widespread corticosubcortical circuits are involved in epileptogenic activity of patients with WS. Epileptiform activity from the primary cortical abnormality stimulates brain stem structures, and interactions of the brain stem with the striatum and cortices induce hypsarrhythmia on EEG. ${ }^{10}$ Persistent or recurrent epileptic activity may have disturbed white matter maturation and led to abnormal findings on DTI in our study. EEG findings during the follow-up period reportedly showed strong correlations with cognitive and motor development. ${ }^{28}$ Our findings suggest that chronic epileptic activity may cause developmental regression by disturbing white matter maturation, though there is the other possibility that preexisting white matter abnormalities might lead to the poor outcome of seizures and EEG findings.

The persistent abnormal findings on DTI at 24 months in children with poor developmental outcome may be multifactorial. Delayed maturation of white matter, microstructural damage by epileptic activities, or the underlying cause of epilepsy may contribute to the reduction in FA values. At 24 months of age, distributions of abnormal findings on DTI corresponded to those of PET hypometabolism, though the abnormal findings on DTI were still widespread and bilateral. Regional PET hypometabolism in patients with WS reportedly suggests focal cortical dysplasia. ${ }^{2}$ Focal cortical dysplasia often involves white matter changes, and a pathologic study has revealed a reduced number of myelinated axons in the white matter. ${ }^{18}$ DTI is reported to show abnormality in the subcortical and deep white matter subjacent to areas of focal cortical dysplasia. ${ }^{20}$ From these observations, the asymmetric abnormal findings on DTI corresponding to areas of PET hypometabolism may represent white matter changes related to the underlying pathology, such as focal cortical dysplasia. In contrast to the reversible FA reduction due to delayed myelination at 12 months of age, abnormal findings on DTI at 24 months of age in patients with poor seizure and developmental outcomes appear to represent persistent microstructural abnormalities. This hypothesis should be proved pathologically or by other imaging techniques such as 7T MR imaging.

Our study has some limitations. First, control subjects were recruited from among patients who had undergone screening scans for CNS abnormalities. Although CNS abnormalities were ruled out clinically, the possibility of underlying CNS lesions cannot be excluded. Second, the ages of patients and controls were not the same. We recruited control subjects with the same mean age as that of patients and used age as a nuisance covariate of no interest on TBSS analysis to reduce the effects of differences in age. However, the effects of the difference in age may not be eliminated completely. Another methodologic limitation is that DTI was performed with 12 directions that were relatively small in number. DTI with more directions and information from the Human Connectome Project (http://humanconnectome.org/) may enable more detailed analysis of white matter fibers. Several clinical factors affect developmental outcome, such as the adverse effects of antiepileptic drugs and the delay of initial treatment. Although we did not use phenobarbital, phenytoin, or topiramate, which were reported to have possible adverse effects on cognitive function, there was the possibility that antiepileptic drugs affect the cognitive function. It has been reported that a delay of initial treatment was a risk factor for poor developmental outcome. ${ }^{29} \mathrm{In}$ our study, only 4 patients had an interval of 2 months or longer from the onset of spasms to initial treatment, and most of the patients had a short treatment lag. Therefore, it is not likely that the delay of treatment caused the abnormal findings on DTI and poor developmental outcome.

\section{CONCLUSIONS}

DTI revealed white matter abnormalities in patients with WS of unknown etiology. These DTI abnormalities correlated with cognitive and motor development, seizure outcome, EEG abnormality, and PET hypometabolism. Serial DTI appears useful for assessing maturational status and detecting underlying pathology. The hypothesis of delayed myelination and microstructural abnormality causing the abnormal findings on DTI needs to be assessed by pathologic correlation, higher resolution neuroimaging, and correlation with therapeutic interventions.

\section{ACKNOWLEDGMENTS}

We thank Drs Shigeki Aoki and Keigo Shimoji from the Department of Radiology at Juntendo University for technical support with image analysis.

\section{REFERENCES}

1. Riikonen R. Epidemiological data of West syndrome in Finland. Brain Dev 2001;23:539-41 CrossRef Medline

2. Chugani HT, Shields WD, Shewmon DA, et al. Infantile spasms, I: PET identifies focal cortical dysgenesis in cryptogenic cases for surgical treatment. Ann Neurol 1990;27:406-13 CrossRef Medline

3. Maeda N, Watanabe K, Negoro T, et al. Transient focal cortical hypometabolism in idiopathic West syndrome. Pediatr Neurol 1993;9: 430-34 CrossRef Medline

4. Maeda N, Watanabe K, Negoro T, et al. Evolutional changes of cortical hypometabolism in West's syndrome. Lancet 1994;343: 1620-23 CrossRef Medline

5. Natsume J, Watanabe K, Maeda N, et al. Cortical hypometabolism and delayed myelination in West syndrome. Epilepsia 1996;37: 1180-84 CrossRef Medline

6. Itomi K, Okumura A, Negoro T, et al. Prognostic value of positron emission tomography in cryptogenic West syndrome. Dev Med Child Neurol 2002;44:107-11 CrossRef Medline

7. Metsähonkala L, Gaily E, Rantala H, et al. Focal and global cortical hypometabolism in patients with newly diagnosed infantile spasms. Neurology 2002;58:1646-51 CrossRef Medline

8. Hamano S, Yoshinari S, Higurashi N, et al. Regional cerebral blood flow and developmental outcome in cryptogenic West syndrome. Epilepsia 2007;48:114-19 CrossRef

9. Natsume J, Maeda N, Itomi K, et al. PET in infancy predicts longterm outcome during adolescence in cryptogenic West syndrome. AJNR Am J Neuroradiol 2014;35:1580 -85 CrossRef Medline

10. Juhász C, Chugani HT, Muzik O, et al. Neuroradiological assessment of brain structure and function and its implication in the pathogenesis of West syndrome. Brain Dev 2001;23:488-95 CrossRef Medline

11. Tsumori M, Inage K. Determination of Psychological Growth for Infants 0-3 Years Old. Tokyo: Dainippon Tosho; 1961

12. Hamano S, Tanaka M, Mochizuki M, et al. Long-term follow-up study of West syndrome: differences of outcome among symptomatic etiologies. J Pediatr 2003;143:231-35 CrossRef Medline 
13. Smith SM, Jenkinson M, Johansen-Berg H, et al. Tract-based spatial statistics: voxelwise analysis of multi-subject diffusion data. Neuroimage 2006;31:1487-505 CrossRef Medline

14. Fosi T, Chu C, Chong WK, et al. Quantitative magnetic resonance imaging evidence for altered structural remodeling of the temporal lobe in West syndrome. Epilepsia 2015;56:608-16 CrossRef Medline

15. Budde MD, Kim JH, Liang HF, et al. Toward accurate diagnosis of white matter pathology using diffusion tensor imaging. Magn Reson Med 2007;57:688-95 CrossRef Medline

16. Song SK, Yoshino J, Le TQ, et al. Demyelination increases radial diffusivity in corpus callosum of mouse brain. Neuroimage 2005;26: 132-40 CrossRef Medline

17. Mukherjee P, Miller JH, Shimony JS, et al. Normal brain maturation during childhood: developmental trends characterized with diffusion-tensor MR imaging. Radiology 2001;221:349-58 CrossRef Medline

18. Shepherd C, Liu J, Goc J, et al. A quantitative study of white matter hypomyelination and oligodendroglial maturation in focal cortical dysplasia type II. Epilepsia 2013;54:898-908 CrossRef Medline

19. Eriksson SH, Rugg-Gunn FJ, Symms MR, et al. Diffusion tensor imaging in patients with epilepsy and malformations of cortical development. Brain 2001;124:617-26 CrossRef Medline

20. Widjaja E, Zarei Mahmoodabadi S, Otsubo H, et al. Subcortical alterations in tissue microstructure adjacent to focal cortical dysplasia: detection at diffusion-tensor MR imaging by using magnetoencephalographic dipole cluster localization. Radiology 2009; 251:206-15 CrossRef Medline

21. Gross DW, Concha L, Beaulieu C. Extratemporal white matter abnormalities in mesial temporal lobe epilepsy demonstrated with diffusion tensor imaging. Epilepsia 2006;47:1360-63 CrossRef Medline

22. Hüppi PS, Maier SE, Peled S, et al. Microstructural development of human newborn cerebral white matter assessed in vivo by diffusion tensor magnetic resonance imaging. Pediatr Res 1998;44:584-90 CrossRef Medline

23. Neil JJ, Shiran SI, McKinstry RC, et al. Normal brain in human newborns: apparent diffusion coefficient and diffusion anisotropy measured by using diffusion tensor MR imaging. Radiology 1998; 209:57-66 CrossRef Medline

24. Hermoye L, Saint-Martin C, Cosnard G, et al. Pediatric diffusion tensor imaging: normal database and observation of the white matter maturation in early childhood. Neuroimage 2006;29:493-504 CrossRef Medline

25. Dubois J, Dehaene-Lambertz G, Perrin M, et al. Asynchrony of the early maturation of white matter bundles in healthy infants: quantitative landmarks revealed noninvasively by diffusion tensor imaging. Hum Brain Mapp 2008;29:14-27 CrossRef Medline

26. Cancelliere A, Mangano FT, Air EL, et al. DTI values in key white matter tracts from infancy through adolescence. AJNR Am J Neuroradiol 2013;34:1443-49 CrossRef Medline

27. Widjaja E, Skocic J, Go C, et al. Abnormal white matter correlates with neuropsychological impairment in children with localizationrelated epilepsy. Epilepsia 2013;54:1065-73 CrossRef Medline

28. Saltik S, Kocer N, Dervent A. Informative value of magnetic resonance imaging and EEG in the prognosis of infantile spasms. Epilepsia 2002;43:246-52 CrossRef Medline

29. Hamano S, Yoshinari S, Higurashi N, et al. Developmental outcomes of cryptogenic West syndrome. J Pediatr 2007;150:295-99 CrossRef Medline 